\title{
Immunological response in coeliac disease is age related
}

\author{
M. Calzado, E. Donat, B. Polo, B. Baena and C. Ribes \\ Pediatric Gastroenterology and Hepatology Section, La Fe Hospital, Valencia, Spain
}

\begin{abstract}
Anti-tissue transglutaminase antibodies (t-TG), mostly IgA class, is considered to be the most specific serological immune response in coeliac disease, and is associated with enteropathy, specific Ig A class antibody against t-TG being found at the intestinal level ${ }^{(1,2)}$.

The objective was to evaluate the serological immune response in coeliac disease according to age by measuring antibodies against the autoantigen, i.e. t-TG and if t-TG can be used as a screening method. A retrospective study was done reviewing the files of all patients who had undergone a small intestinal biopsy in the previous 3 years for diagnostic purposes. IgA t-TG (Varelisa ${ }^{\mathrm{TM}}$, Phadia, cut off 6 IU/ $\mathrm{ml}$ ) and IgA anti-gliadin antibodies (AGA; home method, cut off 0.3 arbitrary units) were determined when the biopsy was performed. In patients with negative IgA t-TG, IgG t-TG (Varelisa ${ }^{\text {TM}}$; Phadia, Freiburg, Germany; cut off $10 \mathrm{IU} / \mathrm{ml}$ ) was determined. A total of 280 patients were included, 160 of them showed Marsh 3 lesions and one of them showed Marsh 2 lesions. All of them were consuming gluten at the time the biopsy was performed. Diagnosis of coeliac disease was established in all of them. No patient had IgA deficiency. All children with t-TG $>100$ (upper detection limit) were classified as Marsh 3. Specificity for AGA was lower than that for t-TG. Eighty-eight patients $(55 \%)$ were $<3$ years of age and sixty-one were $<2$ years of age. Ten of the eighty-eight patients $(11.4 \%)$ were IgA t-TG negative, only one being $>2$ years of age. All ten subjects had elevated IgA AGA and no Ig G class antibodies against t-TG were detected. Clinical symptoms in patients who were t-TG positive and t-TG negative were the same.
\end{abstract}

\begin{tabular}{lcc}
\hline & $<3$ years old $(n$ 88) & $>3$ years old $(n$ 73) \\
\hline Ig A t-TG positive & 78 & 82 \\
Ig A t-TG negative & 10 & 1 \\
IgA AGA positive & 88 & 75 \\
\hline
\end{tabular}

The results show negative $\operatorname{IgA}$ class t-TG is expected in about $10 \%$ of patients with coeliac disease who are $<2$ years of age. Furthermore, no Ig G response is to be expected in those patients. It can be speculated that the immature development of the immune system slowly responding to gluten exposure delays the appearance of autoimmunity. This notion would be in keeping with reports of no IgA t-TG antibodies deposited at the intestinal level in younger patients ${ }^{(3-5)}$. Moreover, the data confirm the response against gliadin is the immunological trigger in coeliac disease.

1. Salmi TT, Collin P, Jarvinen O et al. (2006) Aliment Pharmacol Ther 24, 541-552.

2. Salmi TT, Collin P, Korponay-Szabó IR et al. (2006) Gut 55, 1746-1753.

3. LC Stene, MC Honeyman, EJ Hoffenberg et al. (2006) Am J Gastroenterol 101, 2333-2340.

4. Norris JM, Barriga K, Hoffenberg EJ et al. (2005) JAMA 293, 2343-2351.

5. Tosco A, Maglio M, Paparo F, Colicchio B, Sannino A, Granata V, Rapacciulo L \& Troncone R (2007) Proceedings of the 40th Meeting of the European Society of Paediatric Gastroenterology, Hepatology and Nutrition, Barcelona 2007, Abstr (In the Press). 